\title{
Nano Analysis of Silver Nanoparticles in Commercial Socks
}

\author{
Amy Hemmati*, Keana Scott, Jeff Davis
}

National Institute of Standards and Technology, Surface and Microanalysis Science Division (837), 100 Bureau Drive, Stop 8372, Gaithersburg, MD 20899-8372

*Corresponding author phone: (301)975-8667; email: amy.hemmati@nist.gov

During the last 10 years, the use of silver nanoparticles as sterilizing agents has increased in a number of different consumer products. Silver nanoparticles are able to penetrate through bacterial membranes and react with sulfur and phosphorus based structures, including DNA [1]. Textile companies have dispersed these silver nanoparticles on sock fabric to kill odor causing bacteria. In a recent work, researchers analyzed these socks in sock ashes to confirm the presence of silver nanoparticles and in wash water to examine the possible environmental effects of silver nanoparticles leaching from the fabric during wash cycles [2]. This study suggests that the release of silver during washing could depend on the sock's manufacturing process.

We investigated socks containing silver nanoparticles to verify the presence of silver and characterize the structural details and the distribution patterns of the nanoparticles. Six brands of socks were selected for analysis. The socks were advertised to exploit the antibacterial properties of silver nanoparticles and available over the internet and in stores. Using various types of microscopy and microanalysis techniques, we determined where the silver nanoparticles were located on each sock. First, a few threads were isolated from different areas of each sock (e.g. toe, ankle and heel) and mounted on SEM sample stubs using carbon tape. These threads were then carbon coated to reduce charging during SEM imaging. By exploiting the large difference in average atomic number between the silver nanoparticles and the cotton or synthetic threads, we were able to confirm the presence and identify the locations of silver nanoparticles in the backscattered electron images. XRF spectra of several points on the surface of the socks confirmed the presence silver, as well as zinc and titanium. While the surfaces of some threads were covered in silver nanomaterial, spectra of other threads did not reveal any silver nanoparticles.

Based on these findings, we conclude that electron microscopy and X-ray analysis can be used effectively in identifying and analyzing silver nanoparticles on sock surfaces. Further research is needed to evaluate how the structural details correlate with different leaching rates.

\section{References}

(1) Morones, J., Elechigurra, J., Camacho, A., Holt, K., Kouri, J., Ramirez, J., et al. (2005, August). The bactericidal effect of silver nanoparticles. Nanotechnology, 16, 2346-2353.

(2) Benn, T., Westerhoff, P., (2008, March). Nanoparticle silver released into water from Commercially Available sock fabrics. Environ. Sci. Technol., 42 4133-4139. 

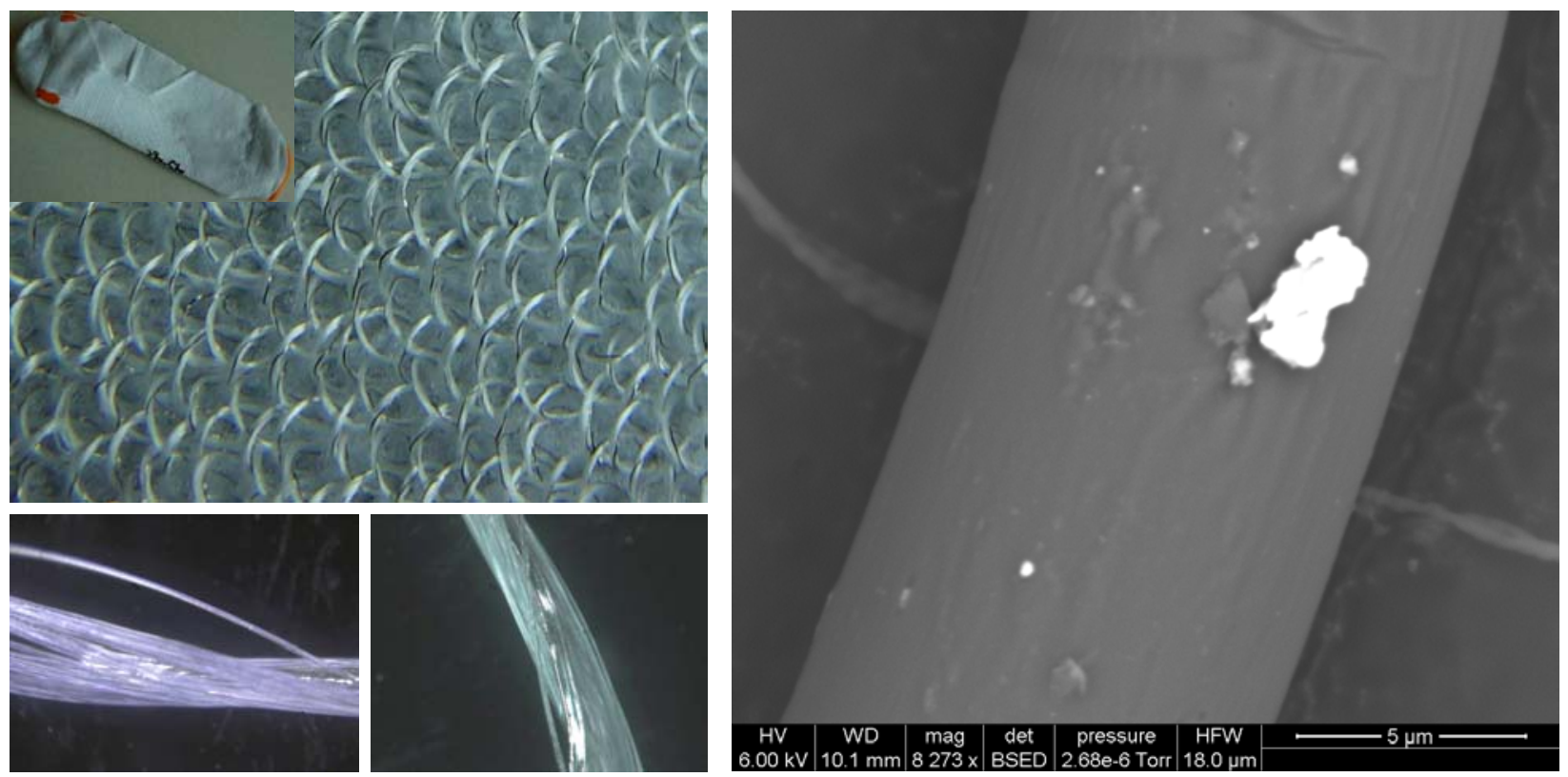

\section{Figure 1: Optical and Electron Microscope Images of Sock Threads}

Top Left: Photograph of sock fibers with inset of full sock. Note the intertwining of light and dark colored fibers.

Bottom Left: Reflected light images of single sock threads at 100x. The silver containing threads are more reflective and opaque.

Right: Micrograph of a sock fiber. The bright spots contain silver material.

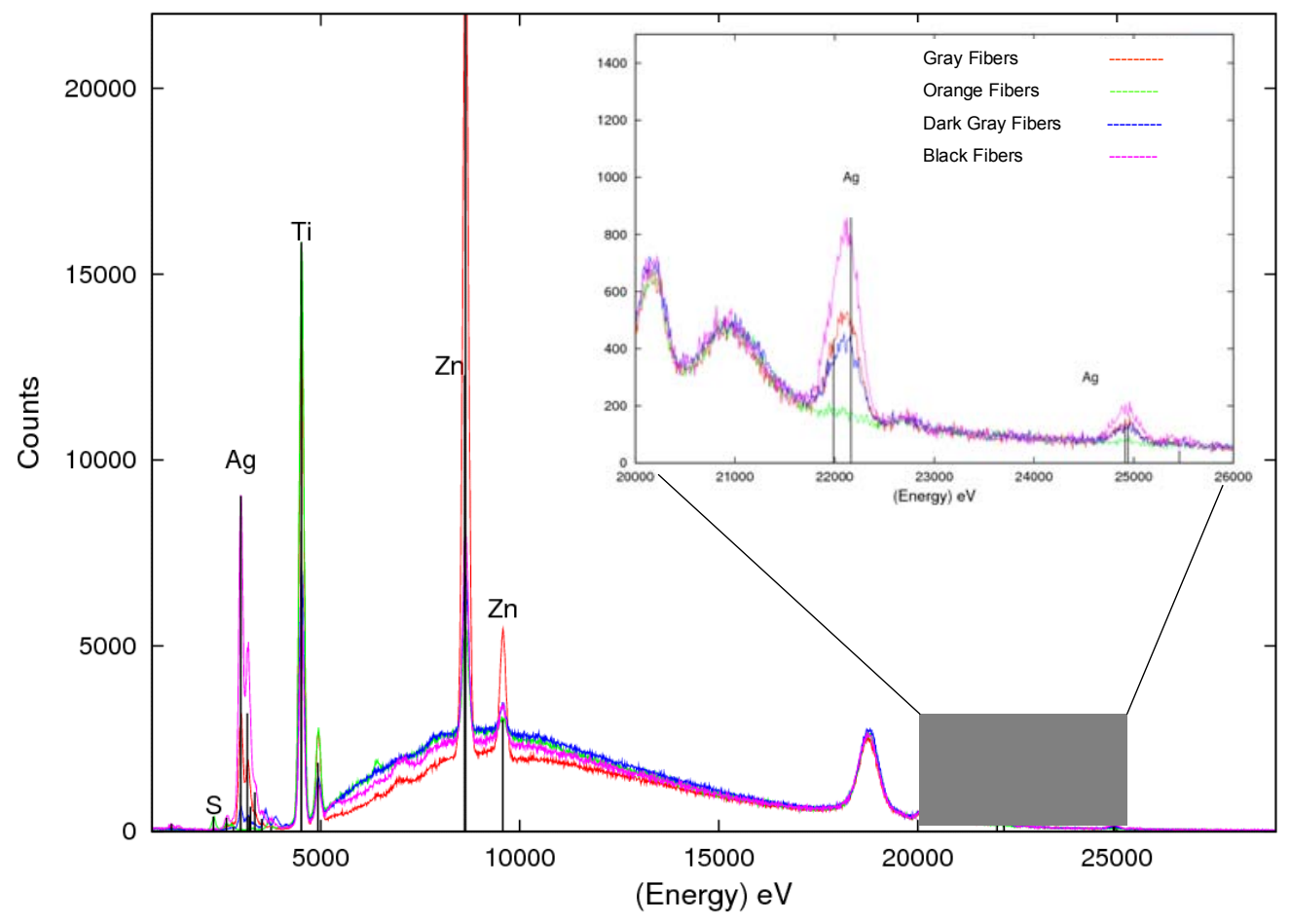

Figure 2: mXRF Spectra at Several Points on the Sock

Milli XRF Spectra from several different fibers found on various areas of the sock. These fibers each contain different amounts of silver material. 